\title{
Amoxicillin Morbilliform Drug Eruption in Pediatric Male with Poor Feeding Treated with Cyproheptadine: A Case Report
}

\author{
Daniel Nguyen, ${ }^{\text {Christina Vo. }}{ }^{2}$
}

\begin{abstract}
Background: Cyproheptadine (CY) is an antihistaminic agent that is commonly used for symptom relief in skin conditions. The most common pattern of cutaneous drug eruption in children is the exanthematous type, with the penicillin family often cited. $\mathrm{CY}$ is also an antiserotoninergic agent with the side effect of appetite stimulation and has been used in children with poor feeding and poor weight gain. The Case: We report a case of a 31-month-old male patient seen in the outpatient setting with a diffuse morbilliform rash after use of amoxicillin for right otitis media. The patient was a post-operative congenital heart disease (CHD) patient, actively being treated with $\mathrm{CY}$ for feeding difficulties and low weight often seen in the CHD population. Amoxicillin was discontinued, while $\mathrm{CY}$ was continued. The patient did not encounter any pruritic symptoms during morbilliform rash, while weight gain of $3.1 \mathrm{~kg}$ occurred over a 9 months period, increasing patient from the 10th to 41 st percentile. Conclusion: A review of studies on CY has shown antiallergic properties in histamine-mediated hypersensitivity reactions, most likely through $\mathrm{H}_{1}$ receptor antagonism. This mechanism may be used to address the pruritic symptoms during type IV T-cell mediated hypersensitivity cutaneous drug eruptions. CY also possesses 5 -HT receptor antagonist properties with demonstrated ability to increase appetite in poor feeding pediatric patients. CY was successfully used for this purpose in our CHD patient.
\end{abstract}

Key Words: Amoxicillin; Exanthema; Cyproheptadine; Heart Defects, Congenital; Appetite (Source: MeSH-NLM).

\section{Introduction}

Pediatric drug-related cutaneous reaction patterns are numerous, but can generally be classified into five types: exanthemata, fixed drug eruptions, urticarial eruptions, serum sickness-like reactions, and photosensitive eruptions. ${ }^{1}$ The most common pattern of cutaneous drug eruption in children is the exanthematous type. ${ }^{1}$ The penicillin family is often implicated, with amoxicillin a frequent culprit.

Drug reactions can occur in patients with co-morbidities, complicating the management. One potential comorbidity is failure to thrive, which may indicate the need for an appetite stimulant. ${ }^{2}$ Cyproheptadine (CY) is an antihistaminic and antiserotoninergic agent that has United States Food at Drug Administration (FDA) approval for indications of allergic conditions while offering a beneficial side effect of appetite stimulation in various conditions.3-4 These conditions include anorexia nervosa, cystic fibrosis, asthma, malignancies, stimulant-induced weight loss, malnourished, and short stature.2,5 We present a patient being chronically treated with $\mathrm{CY}$, who continued therapy to address a separate acute condition. The use of CY is reviewed for our patient's comorbidities, an amoxicillin adverse drug reaction and feeding difficulties in repaired congenital heart disease (CHD) patient.

\section{The Case}

We evaluated a 31-month-old Asian male in the clinic with a chief complaint of rash. The rash onset began a day before with no particular pattern (i.e., cephalocaudal), and described as non-pruritic, nontender, worsening rash all over the body. Parents were unaware of any previous penicillin exposure while reporting up-to-date immunization status. Parents deny history of fevers, chills, cough, nausea, vomiting,

Key Poin Is:
- Cyproheptadine is an antihistaminic and antiserotoninergic agent
that has US FDA approval for indications of allergic conditions, with
beneficial side effects of appetite stimulation.
- Cyproheptadine is often used for symptomatic relief in pediatric
patients for penicillin-induced exanthematous cutaneous drug
eruption, a type IV T-cell mediated hypersensitivity. While using
cyproheptadine, our patient did not have any pruritic symptoms
during the morbilliform rash, from onset through resolution.
- Cyproheptadine has been used in feeding programs for children
with feeding difficulties and poor growth. The post-operative
congenital heart disease patient with a history of poor weight gain,
increased in weight from $10.25 \mathrm{~kg}$ ( $10^{\text {th }}$ percentile) to $13.35 \mathrm{~kg}\left(41^{\text {th }}\right.$
percentile) during cyproheptadine use.

diarrhea, allergies, or any other previous rash. Family history not significant for allergies or similar rashes. Recent upper respiratory infection of acute otitis media (AOM) was diagnosed in the patient eight days earlier, but no recent history of pharyngitis noted. Past medical and surgical history involved CHD diagnosed at 4 months of age, specifically atrioventricular canal defect, hypoplastic aortic arch, and patent ductus arteriosus. The patient was hospitalized at the time of discovery for medical management of heart failure, pulmonary overload, and failure to thrive (admission weight $5.3 \mathrm{~kg}[2.3$ percentile, $\mathrm{z}$-score $=-2.0, \mathrm{CDC}]$ ) (Figure 1 ) with nutrition and diuresis optimized in anticipation of surgical repair. Complete repair performed at 6 months of age with no major issues in the operation room. Post-surgical complications developed for hypomobile left vocal cord, oral aversion, and poor weight gain.

\footnotetext{
${ }^{1}$ University of North Texas Health Science Center, Fort Worth, Texas, USA

${ }^{2}$ Encompass Health Rehabilitation Hospital The Vintage, Houston, Texas, USA
}

About the Author: Daniel Nguyen is currently a fourth-year medical student at the University of North Texas Health Science Center, Texas College of Osteopathic Medicine, in Fort Worth, TX, USA. He is also a licensed pharmacist and certified public accountant in the state of Texas. 
Medical interventions for low weight was initiated at 11 months of age (weight of $7.65 \mathrm{~kg}$ [0.4 percentile, z-score $=-2.7, C D C]$ ) with nutrition management, and consultations for dietician, gastroenterology, speech therapy, physical therapy, and occupational therapy. At 19 months of age, weight gain progressed to $9.84 \mathrm{~kg}$ (3.4 percentile, $\mathrm{z}$-score $=-1.8$, CDC) but increases faded 3 months later (22-months of age) at a weight of $10.25 \mathrm{~kg}$ (3.2 percentile, $\mathrm{z}$-score $=-1.8, \mathrm{CDC}$ ). At this point, the decision was made to start oral cyproheptadine. Medications at time of current clinic visit included amoxicillin ( $7 \mathrm{ml}$ of a $400 \mathrm{mg} / 5 \mathrm{ml}$ suspension, twice a day) on day 9 of a 10-day course for recent AOM and $\mathrm{CY}(2.1 \mathrm{ml}$ of a $2 \mathrm{mg} / 5 \mathrm{ml}$ syrup, twice a day) for feeding difficulties. Besides current amoxicillin treatment regimen, no previous penicillin antibiotics noted in medical records. Vital signs at current visit showed temperature of 37.0 degrees Celsius, heart rate of 116 beats per minute, respiratory rate of 26 breaths per minute, and a recorded weight and height of $13.35 \mathrm{~kg}$ ( 41 percentile, $z$-score $=-0.2, C D C$ ), and $91.44 \mathrm{~cm}$ (44 percentile, $\mathrm{z}$-score $=-0.2, \mathrm{CDC}$ ), respectively, (Figure 1 and Figure 2 ).

Figure 1. Patient Weight-For-Age Clinical Growth Chart (CDC) and Initiation of Cyproheptadine. *Initiation of Cyproheptadine at 22 Months of Age.

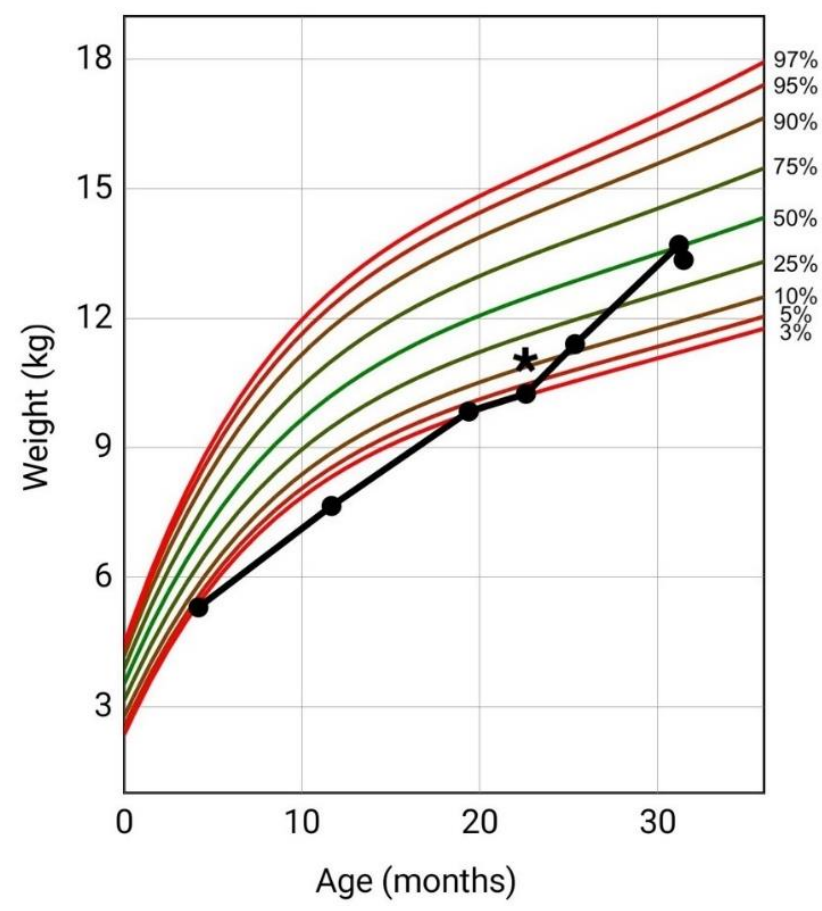

Physical exam showed a well-appearing child in no acute distress. Lungs were clear to auscultation. Heart was normal sinus rhythm with no murmurs identified. Oral exam showed moist mucous membranes with no mucocutaneous lesions or tonsillar exudates. Eyes were bilaterally negative for conjunctivitis. Nasal passages were patent, pink and moist. Ear inspection demonstrated pearly grey, mobile, nonbulging tympanic membranes bilaterally. Supple neck with no adenopathy appreciated. Abdomen exam observed no organomegaly. Skin findings revealed morbilliform rash consisting of round erythematous macule and papule lesions, ranging from 1 to 5 millimeters in diameter, distributed bilaterally on the head, trunk, and extremities, with several confluent patches (Figure 3 and Figure 4).

No bullous lesions or desquamation was noted. Differential diagnosis included measles, rubella, scarlet fever, infectious mononucleosis associated amoxicillin rash, and unlikely, Kawasaki disease, anaphylaxis, and Stevens-Johnson syndrome - toxic epidermal necrolysis.
Figure 2. Patient Length-For-Age Clinical Growth Chart (CDC) and Initiation of Cyproheptadine. *Initiation of Cyproheptadine at 22 Months of Age.

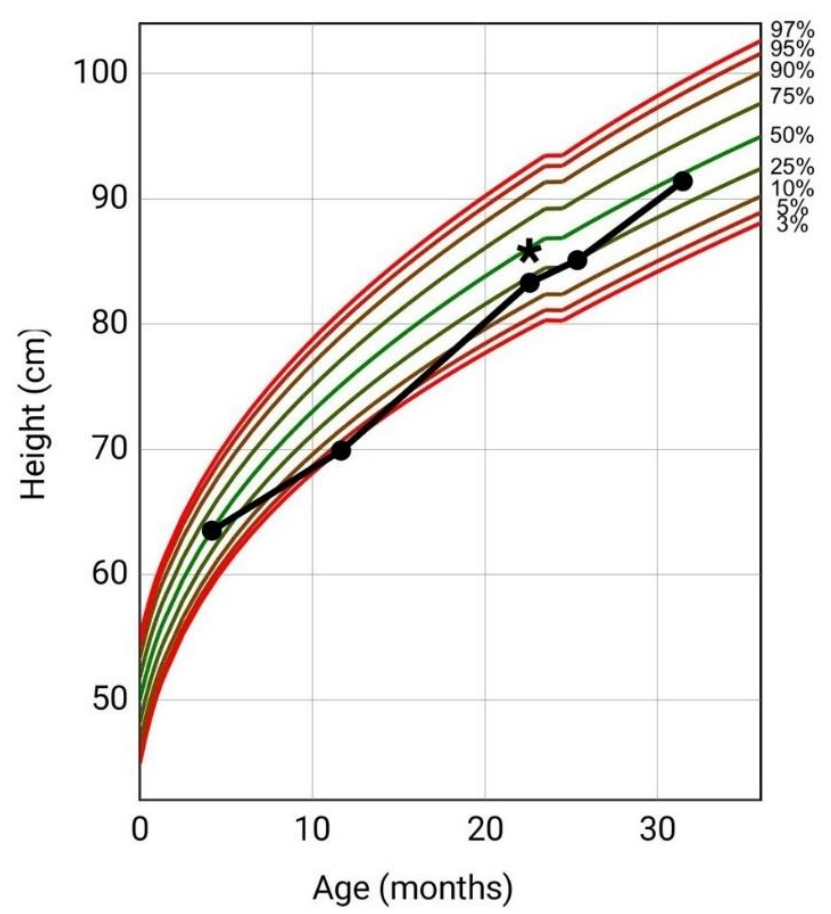

History and physical exam, including timeline of amoxicillin administration, lead to a clinical diagnosis of adverse reaction to penicillin. Management involved consideration for discontinuation of medications. Amoxicillin was stopped due to high suspicion as the causative agent of the rash. Further antibiotic treatment deemed unnecessary as resolution of AOM was noted per physical exam. With a low index of suspicion for $\mathrm{CY}$ as the underlying etiology of the rash, the decision to continue $\mathrm{CY}$ was made. CY outpatient therapy was maintained for management of the dermatologic symptoms, including itch while sustaining enhanced appetite stimulation and weight gain effect. Patient's parents were in agreement to therapeutic plan as they reported good compliance with $\mathrm{CY}$, positive response in terms of improved weight gain and absence of pruritic symptoms. Weight at current encounter was a $1.95 \mathrm{~kg}$ increase from the time of $\mathrm{CY}$ initiation. Per discussion with patients at a subsequent unrelated encounter, rash continued for 2-3 days after discontinuing amoxicillin and cleared approximately a week later.

Figure 3. Morbilliform Rash on Patient's Ventral Trunk.

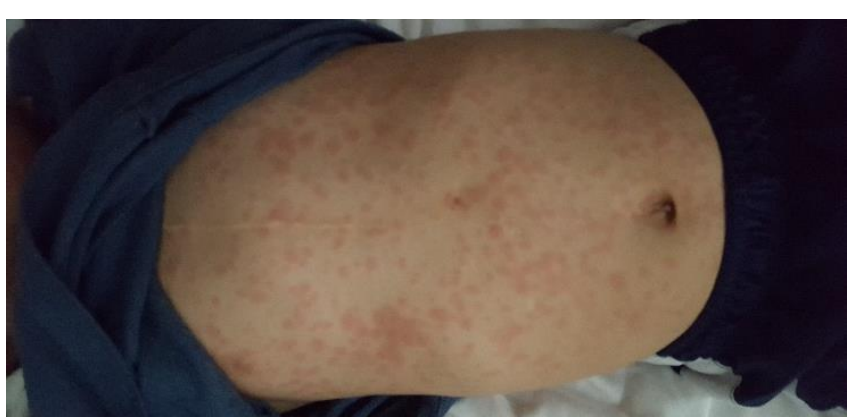


Figure 4. Morbilliform Rash on Patient's Dorsal Trunk.

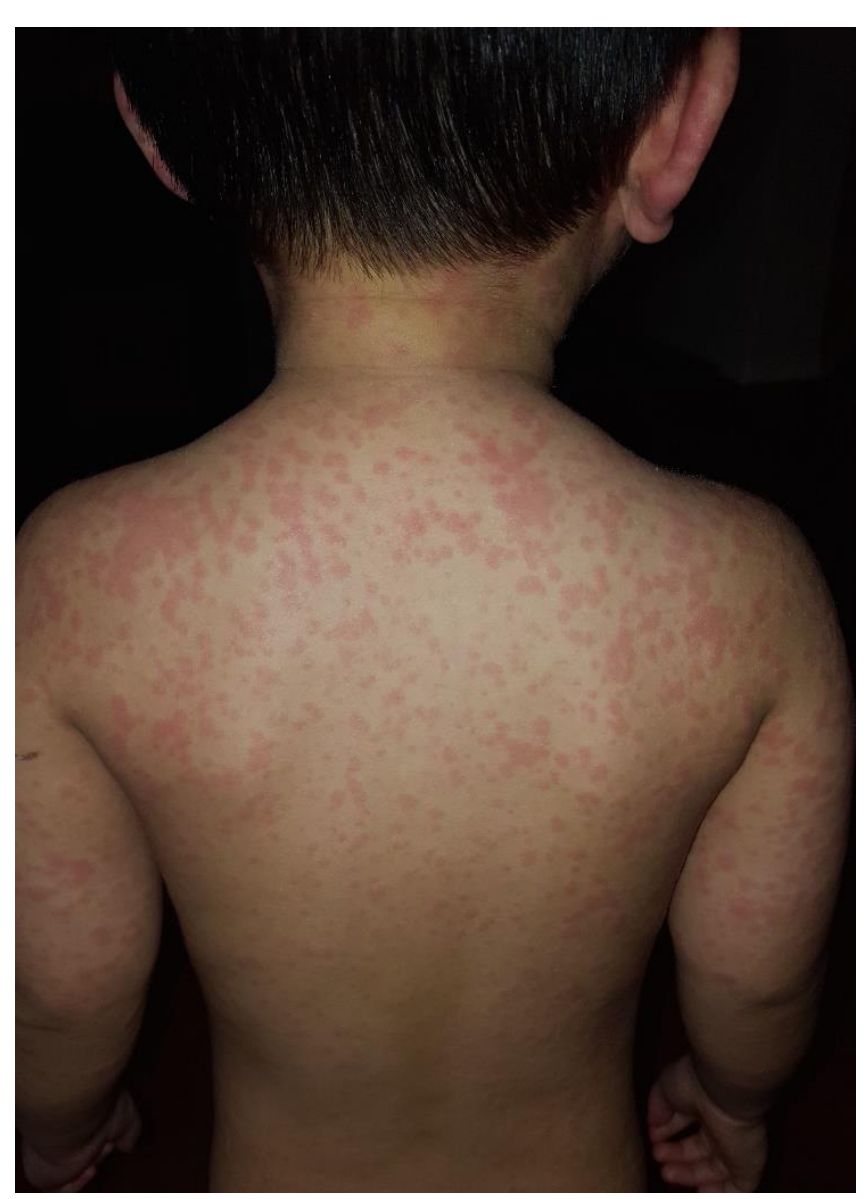

\section{Discussion}

Cyproheptadine is a medication that has antiallergic and orexigenic properties and was utilized in this pediatric patient with an adverse reaction to amoxicillin and feeding difficulties subsequent to postoperative CHD repair.

A recent review of evidence for $C Y$ 's antiallergenic effects in children by De Bruyne et al examined two small prospective comparative studies. 3 Six children in a double-blind, cross-over study showed CY to be similar in effectiveness to ketotifen for primary acquired cold urticarial clinical symptoms and ice cube test. 3 However, a randomized open-label study showed loratadine to be superior to $\mathrm{CY}$ in treating mite-induced allergic rhinitis. 3 Neither study had a placebo control group to compare with CY.3 The studies above pertain to CY's antiallergenic effects in pediatric patients that are effective in disease states with pathophysiology involving mast cell degranulation leading to histamine release (i.e., type I IgE-mediated hypersensitivity reactions). 3 The suspected amoxicillin-induced morbilliform rash, with delayed onset and no associated antibody-mediated cytotoxic effects or immunocomplex mediated syndromes, was classified as a type IV delayed T-cell mediated hypersensitivity reaction. ${ }^{6}$ Immunohistochemical studies of type IV reactions in the skin have found mononuclear cell infiltrates composed mainly of T cells, CD4 predominating over CD8, with additional neutrophils, eosinophils, macrophages, and keratinocytes involvement. ${ }^{6}$ In particular, type IV maculopapular exanthema have shown an increase in eosinophils in the papillary dermis. ${ }^{6}$ However, there is nothing in the literature that reports on CY's effectiveness in amoxicillin mediated type IV hypersensitivity reactions. ${ }^{6}$ Cyproheptadine has well documented $\mathrm{H}_{1}$ antihistaminic properties, but no evidence exists for non-H1-mediated anti-inflammatory properties, which has been seen in other antihistamines agents.7-8

Still, antihistamines are commonly used in the management of type IV adverse drug reactions for dermatological symptoms. ${ }^{9}$ A likely benefit is the antihistamines sedative effects leading to a reduction in itch, especially at night. ${ }^{10-11}$ The natural course of an aminopenicillin rash is variable, with resolution of rash reported 7-15 days after therapy cessation. ${ }^{12}$ Our patient's rash continued for approximately 10 days after amoxicillin withdrawal, consistent with normal rash resolution. Additional consideration for an unclear diagnosis of a rash that may be a type I IgE-mediated hypersensitivity, could benefit from CY and it's FDA indication as adjunctive therapy, in addition to standard measures. 4

In regard to off-label appetite stimulant evidence found by De Bruyne et al for $\mathrm{CY}$ in pediatric patients, there were two retrospective studies. 3 A retrospective chart review found 82 children with low appetite and poor growth who took CY regularly, where $96 \%$ of parents reported a positive change in mealtime and feeding behaviors. ${ }^{2-3}$ Additionally, significant improvement in mean weight-to-age Z scores was observed after starting $C Y .2-3 \mathrm{~A}$ retrospective open-label study for the efficacy of $\mathrm{CY}$ in dyspeptic children showed positive overall response in $55 \%$ of patients. 3 A search of the medical literature did not reveal any reported cases of $C Y$ use in a post-operative CHD patient. CHD patients can have increased energy and nutrient needs, complicated by feeding difficulties. ${ }^{13}$ As our patient had a multi-disciplinary team addressing nutrition, development/behavioral, psychosocial issues over a course of a year with failure to reach catch-up growth, adjunctive therapy in the form of $\mathrm{CY}$ was initiated before considering escalating management to a pediatric feeding program. ${ }^{2}$ Cyproheptadine's antiserotoninergic properties is thought to play a major role in appetite stimulation, though the mechanism is not well understood. A hypothesis for the mechanism of action include antihistaminic and antiserotogenic receptors at the ventromedial hypothalamus and $C Y$ influence on growth hormone and insulin-like growth factor axis. ${ }^{2}$ Either or both of these mechanisms could play a role in increasing appetite and causing weight gain in post-operative CHD patients.

Cyproheptadine has been reported as a safe medication in the pediatric population.2,5 Aside from expected increased appetite, reported adverse effects included self-limited somnolence, irritability, and abdominal pain that often resolves after the first few days of therapy..$^{2-3,5}$ One case report of CY toxicity in a 5 -year-old female described signs and symptoms consistent with anticholinergic toxicity, urinary incontinence, and tachycardia. ${ }^{14}$ Our patient had no reported adverse effects from the medication.

Limitations for use of $\mathrm{CY}$ in cutaneous drug reactions include patient presenting for a rash that is asymptomatic, exposing the patient to unnecessary drug risks. Limitations of weight gain in post-operative repaired CHD revolves around the difficulty in attributing weight gain to medication opposed to the natural course of improvement after correction of the defect. As CY has been well-tolerated in studies with rare adverse events, the clinician should carefully consider the risk versus the benefits of the medication. ${ }^{2-3,5}$

In conclusion, this case report reviews studies of $\mathrm{CY}$ as an antiallergic and orexigenic agent and $C Y$ 's application to a post-operative CHD patient with new-onset amoxicillin morbilliform drug eruption in the background of poor weight gain. Cyproheptadine's $\mathrm{H}_{1}$ and 5 -HT receptor antagonist properties are the mechanism's most strongly supporting CY's use in histamine mediated hypersensitivity reactions and appetite stimulation. We suspect $C Y$ may provide some benefit specifically for delayed type IV T-cell mediated hypersensitivity drug reactions and increased feeding in CHD patients. Clinicians can utilize the dual mechanisms of action in their pediatric patients for various allergic indications and/or difficulty feeding and poor weight gain. 


\section{References}

1. Shin HT, Chang MW. Drug eruptions in children. Curr Probl Pediatr. 2001 Aug;31(7):207-34.

2. Sant'Anna AM, Hammes PS, Porporino M, Martel C, Zygmuntowicz C, Ramsay M. Use of cyproheptadine in young children with feeding difficulties and poor growth in a pediatric feeding program. J Pediatr Gastroenterol Nutr. 2014 Nov;59(5):674-8.

3. De Bruyne P, Christiaens T, Boussery K, Mehuys E, Van Winckel M. Are antihistamines effective in children? A review of the evidence. Arch Dis Child. 2017 Jan;102(1):56-60.

4. Cyproheptadine hydrochloride [package insert]. Sellersville, PA: Teva Pharmaceuticals USA. 2009.

5. Couluris M, Mayer JL, Freyer DR, Sandler E, Xu P, Krischer JP. The effect of cyproheptadine hydrochloride (periactin) and megestrol acetate (megace) on weight in children with cancer/treatment-related cachexia. J Pediatr Hematol Oncol. 2008 Nov;30(11):791-7.

6. Torres MJ, Blanca M. The complex clinical picture of beta-lactam hypersensitivity: penicillins, cephalosporins, monobactams, carbapenems, and clavams. Med Clin North Am. 2010 Jul; $94(4): 805-20$, xii.
7. Fadel R, David B, Rassemont R, Herpin-Richard N, Borgnon A, Rihoux JP. Eosinophil infiltration: effects of $\mathrm{H}_{1}$ antihistamines. J Am Acad Dermatol. 1991 Jun;24(6 Pt 2):10946.

8. Vallero S, Mondino A, Farinasso L, Ansaldi G, Davitto M, Ramenghi U. Successful Use of Antihistamines in Severe Hypereosinophilia. Pediatr Rep. 2012 Jun 22;4(3):e26.

9. Riedl MA, Casillas AM. Adverse drug reactions: types and treatment options. Am Fam Physician. 2003 Nov 1;68(9):1781-90.

10. Carder KR. Hypersensitivity reactions in neonates and infants. Dermatol Ther. 2005 Mar-Apr;18(2):160-75.

11. Denman ST. A review of pruritus. J Am Acad Dermatol. 1986 Mar;14(3):375-92.

12. Romano A, Quaratino D, Papa C, Di Fonso M, Venuti A. Aminopenicillin allergy. Arch Dis Child. 1997 Jun; 76(6):513-7.

13. Roman B. Nourishing little hearts: nutritional implications for congenital heart defects. Pract Gastroenterol. 2011,8:11-32.

14. McGovern T, McNamee J, Marcus S, Kashani J. When Too Much Is Enough: Pediatric Cyproheptadine Overdose with Confirmatory Level. Clin Pract Cases Emerg Med. 2017 Jul 6;1(3):205-207.

\section{Acknowledgments}

None.

Conflict of Interest Statement it Funding

The authors have no funding, financial relationships or conflicts of interest to disclose.

Author Contributions

Conception and design of the work/idea: DN, CV. Collection of data/obtaining results: DN, CV. Analysis and interpretation of data: DN, CV. Writing the manuscript: DN, CV. Critical revision of the manuscript: DN, CV. Approval of the final version: DN, CV. Contribution of patients or study material: DN, CV. Administrative or technical advice: DN, CV.

Cite as:

Nguyen D, Vo C. Amoxicillin Morbilliform Drug Eruption in Pediatric Male with Poor Feeding Treated with Cyproheptadine: A Case Report. Int J Med Students. 2018 May-Aug;6(2):71-74. 